\section{Marcadores de I nflamação em Pacientes Com Diabetes Mellitus Tipo 1}

\author{
RESUMO
}

Para avaliar a resposta inflamatória, representada pelas proteínas de fase aguda, estudamos 48 pacientes com diabetes tipo 1 (DM1) sem


doença] e 66 indivíduos sem DM, pareados quanto ao sexo, idade e estadiamento puberal (critérios de Tanner). Foram dosadas proteína C reativa (PCR), $\alpha$ l-glic oproteína ácida ( $\alpha-1 \mathrm{GPA}$ ) e fibrinogênio, por imunoturbidimetria. A taxa de excreção de albumina (EUA) foi determinada por RIE, em amostra de urina de 10h, definindo-se nomoalbuminúria como duas taxas de EUA $<20 \mu \mathrm{g} / \mathrm{min}$. Pa cientes com DM1 foram a valiados quanto à presença de retinopatia por oftalmoscopia indireta. No DM1 os níveis de PCR $[0,23(0,01-2,90)$ vs. $0,14(0,01-2,41) \mathrm{mg} / \mathrm{dl}, \mathrm{p}=$ $0,0172$ ] e de $\alpha 1-G P A$ [53,5 (37-115) vs. 40 (19-78) $\mathrm{mg} / \mathrm{dl}, \mathrm{p}<0,0001]$ foram maiores quando comparados aos sem DM. Não houve diferença em relação a o fibrinogênio. Na regressão linear múltipla em stepwise, tendo a $\alpha 1-G P A$ como va rável dependente, as va ráveis independentes associadas e preditoras foram a HbAlc $\left(r^{2}=0,26 ; p<0,05\right)$ e a glicemia $\left(r^{2}=\right.$ $0,26 ; p<0,05)$; tendo a $P C R$ e o fibrinogênio como variáveis dependentes, nenhuma variável independente foi signific ativa. Na correlação de Pearson, a PCR correlacionou-se com HbAlc $(r=0,18 ; p=0,05)$. Concluímos que a PCR e $\alpha 1$-GPA estã o aumentadas no DM 1 , independente da presença da mic roa lbuminúria, retinopatia e doença macrovascular c línica. Estudo prospectivo será necessá io para estabelecermos o valor preditivo destes marcadores na evolução para complicações crônicas mic ro e macrovasc ulares. (Arq Bras Endoc rinol Metab 2004;48/2:253-260)

Descritores: Resposta inflama tória; Proteínas de fase aguda; Proteína C reativa; Fib rinogênio; Alfa-1 glic oproteína ácida; Diabetes tipo 1

\begin{abstract}
Markers of Inflammation in Type 1 Diabetic Patients.

To evaluate markers of inflammation, we studied 48 patients with type 1 diabetes [DM1, 23F:25M, 19.9 \pm 9.8 years and duration of DM of 5 (1-21) years] and 66 non-DM subjects, matched for sex, age, and stages of puberty according to Tanner. C-reactive protein (CRP), $\alpha 1$-acid glycoprotein (AGP) and fibrinogen were measured by turbidimetric immunoassay and urinary albumin excretion rate (AER) was determined in timed ovemight urine samples by RIA. Mic roalbuminuria was defined when two out of three urine samples had AER ranging 20-200 $\mu \mathrm{g} / \mathrm{min}$. Retinopathy was evaluated by indirect ophthalmoscopic in DM patients. The CRP and AGP levels were higher in DM1 patients as compared to controls, respectively [0.23 (0.01-2.90) vs. $0.14(0.01-2.41) \mathrm{mg} / \mathrm{dl}, \mathrm{p}=0.0172]$ and [53.5 (37$115)$ vs. 40 (19-78) mg/dl, $p<0.0001]$. Fibrinogen levels were not different between both groups. Stepwise multiple regression a nalysis showed that $\mathrm{HbAlc}$ and plasma glucose were the independents predictive variables of $A G P$, respectively $\left(r^{2}=0.26 ; p<0.05\right.$ and $\left.r^{2}=0,29 ; p<0,05\right) ; C R P$ and fibrinogen did not correlate significantly with the independents variables. PCR correlate with HbAlc $(r=0.18 ; p=0.05)$ by Pearson's correlation. In conclusion, CRP and AGP were higher in DM1 patients, without mic roal-
\end{abstract}

artigo original

\author{
Laura J. Piccirillo \\ Maria de F.R. Gonçalves \\ Eliete L.S. Clemente \\ Marília de B. Gomes
}

\author{
Disciplina de Diabetese \\ $M$ etabologia, D epartamento \\ de Medicina I nterna da \\ U niversidade do Estado do \\ Rio de Janeiro - UERJ, \\ $\mathrm{R}$ io deJ aneiro, RJ.
}

R ecebido em 12/02/03

R evisado em 23/09/03 A cei to em 29/09/03 
buminuria, retinopathy and clinical macrovascular disease. Prospective studies must be addressed to determine the influence of AGP and CRP in the development of chronic complications. (Arq Bras Endocrinol Metab 2004;48/2:253-260)

Keywords: Inflammation; Acute-phase response; Creactive protein; Fibrinogen; $\alpha-1$ acid glycoprotein; Type 1 diabetes

0 Diabetes mellitus CONSTITUI um fator de risco independente para o desenvolvimento da aterosclerose (1). Os mecanismos responsáveis pelo risco aumentado da aterosclerose ainda são pouco compreendidos, mas numerosas observações suportam a teoria de que a inflamação crônica esteja envolvida no processo de progressão das alterações vasculares $(2,3)$. Estudos recentes têm demonstrado que a aterosclerose não é simplesmente uma doença de depósito de lipídeos e que a inflamação tem papel fundamental na iniciação, progressão e desestabilização do ateroma (4).

U m marcador da atividade inflamatória é o aumento na circulação das proteínas de fase aguda produzidas pelo fígado, como a proteína $C$ reativa (PCR), a alfa 1 glicoproteína ácida e o fibrinogênio. A PCR é uma das proteínas de fase aguda mais sensíveis, cuja concentração aumenta significativamente durante uma inflamação aguda (5). 0 interesse no estudo desta proteína tem crescido nos últimos anos, uma vez que pequenos aumentos em sua produção estão associados a um aumento no risco de doença cardiovascular em pacientes com angina pectoris (6) e também em indivíduos saudáveis (7). A alfa 1 glicoproteína ácida ( $\alpha 1$-GPA) é uma proteína de fase aguda do tipo 1, cuja secreção é regulada pela interleucina 1 (IL-1), interleucina 6 (IL - 6 ) e do fator de necrose tumoral- $\alpha$ (TNF- $\alpha$ ), importantes mediadores da resposta inflamatória e imune (8).

Também tem sido proposto que o estado de hipercoagulabilidade encontrado no diabetes contribua, pelo menos em parte, para o processo de ateroesclerose. $D$ entre os vários fatores hemorreológicos, o fibrinogênio elevado como fator de risco no diabetes tem merecido especial atenção (9). O fibrinogênio é uma glicoproteína, componente fundamental da cascata de coagulação. Elevações em sua concentração plasmática podem ocorrer em reposta a inflamações, infecções, traumas e estresse emocional. 0 tabagismo, o IMC e a idade também influenciam suas concentrações (10). Vários estudos têm demonstrado que o fibrinogênio é um importante e independente fator de risco para doença cardiovascular (10). 0 aumento de seus níveis representaria um estado de hipercoagulabilidade sangüínea (11), que contribuiria, possivelmente, para a aterosclerose acelerada nestes pacientes $(12,13)$.

A concentração do fibrinogênio está freqüentemente elevada no diabetes, particularmente no tipo 2 e nos pacientes com complicações vasculares. Entretanto, mesmo nos diabéticos tipol e naqueles sem complicações vasculares, os níveis do fibrinogênio têm se mostrado elevados (9). Os diabéticos com complicações microvasculares também têm maiores níveis de fibrinogênio (10) e o controle glicêmico também parece estar relacionado às suas concentrações (14). O s diabéticos podem apresentar níveis elevados da PCR e da $\alpha$ l-G PA, além do fibrinogênio. A alta incidência de aterosclerose nos pacientes diabéticos faz com que a identificação dos pacientes com maior risco seja de relevante importância. Embora o aumento das proteínas de fase aguda circulantes tenha sido reportado no diabetes mellitus $(2,15,16)$, as concentrações destas proteínas, especificamente no diabetes do tipo 1 , ainda não foram estudadas sistematicamente.

0 presente estudo teve como objetivo avaliar a resposta inflamatória, através de determinações sangüíneas da PCR, $\alpha 1$-GPA e fibrinogênio, nos diabéticos tipol sem complicações micro e macrovasculares, acompanhados no ambulatório de diabetes do H ospital U niversitário Pedro Ernesto, comparando-os a um grupo de indivíduos não diabéticos.

\section{PACIENTES E MÉTODOS}

Foram estudados 48 pacientes com DM 1, sendo 6 crianças, 15 adolescentes e 27 adultos, 23 (48\%) do sexo feminino e 25 (52\%) do sexo masculino, classificados de acordo com os critérios da Associação Americana de Diabetes (ADA) (17) e regularmente acompanhados no ambulatório de Diabetes do $\mathrm{H}$ ospital U niversitário Pedro Ernesto da U niversidade Estadual do Rio de J aneiro; com idade de 19,9 $\pm 9,8$ anos, idade ao diag-

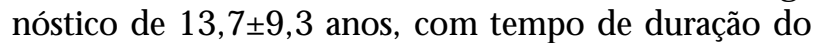
diabetes de 5 (1-21) anos, em uso de 0,8 $\pm 0,4$ unidades de insulina por kilograma de peso por dia (U/ Kg/ dia) e ausência de retinopatia, microalbuminúria e doença macrovascular clínica. A população não diabética constituiu-se de 66 indivíduos não diabéticos, pareados quanto ao sexo, idade e estadiamento puberal pelos critérios de Tanner (18); sendo 8 crianças, 18 adolescentes e 40 adultos, 40 (61\%) do sexo feminino e 26 (39\%) do sexo masculino, com idade de $23,1 \pm 10,9$ anos. Os dados demográficos das populações estudadas estão descritos na tabela 1. 
Tabela 1. Dados clínicose demográfic os dos grupos não diabétic os e diabéticos.

\begin{tabular}{lcc}
\hline Variáveis & $\begin{array}{c}\text { Não Diabéticos } \\
n=66\end{array}$ & $\begin{array}{c}\text { Diabéticos } \\
n=48\end{array}$ \\
\hline Sexo (M/F) & $26 / 40$ & $25 / 23$ \\
Idade (anos) & $23,1 \pm 10,9$ & $19,9 \pm 9,8$ \\
Idade diagnóstico (anos) & - & $13,7 \pm 9,3$ \\
Estadiamento Puberal (1/2/5) & $8 / 18 / 40$ & $6 / 15 / 27$ \\
Dose insulina (U/Kg) & - & $0,8 \pm 0,4$ \\
Duração diabetes (anos) & - & $5,0(1,0-21,0)$ \\
\hline
\end{tabular}

M: masc ulino; F: feminino; 1: crianças; 2: adolescentes; 5: adultos.

Todos os indivíduos foram submetidos a uma avaliação clínico-laboratorial, sendo apurados dados relativos à história de infecção recente, com aferição da pressão arterial e da temperatura axilar e a uma avaliação metabólica e bioquímica, determinando-se a glicemia, H bAlc, fibrinogênio, PCR e $\alpha 1-G P A$.

A pressão arterial foi determinada com 0 paciente em posição deitada após repouso de cinco minutos, em intervalos de cinco e dez minutos e sentada após cinco minutos nesta posição. U tilizou-se um esfignomanômetro de coluna de mercúrio padronizado e calibrado e manguitos de tamanho recomendado para cada faixa etária. A pressão diastólica foi determinada pelo desaparecimento dos sons de Korotkoff fase 5 para os indivíduos com idade superior a 12 anos e pela fase 4 para aqueles menores de 12 anos (19). Foram calculadas, a partir destes dados, as médias aritméticas das três aferições realizadas, respectivamente, nas três visitas ao $\mathrm{H}$ ospital, obtendo-se as médias da pressão arterial sistólica e diastólica.

0 indivíduo foi considerado hipertenso quando a média das três aferições de pressão arterial sistólica (PAS) em posição supina aos cinco minutos foi superior ou igual a $140 \mathrm{mmH} \mathrm{g} \mathrm{e/} \mathrm{ou} \mathrm{a} \mathrm{de} \mathrm{pressão} \mathrm{arterial} \mathrm{diastólica}$ (PAD) foi superior ou igual a $90 \mathrm{mmH} g$ para os adultos ou pelo uso de medicação anti-hipertensiva (20). N as crianças e adolescentes, foi considerada hipertensão arterial quando a média das três aferições de PAS e/ ou PAD esteve acima dos níveis de pressão arterial sistólica e diastólica para os percentis $90^{\circ}$ e $95^{\circ}$ de pressão sangüínea para idade e sexo por percentis de altura (19) ou pelo uso de medicação anti-hipertensiva (20).

Todos os pacientes colheram urina noturna da seguinte forma: às 20 horas do dia anterior à ida ao H ospital, os pacientes foram orientados a urinar e desprezar esta amostra. Iniciou-se, então, a coleta da urina noturna, onde todas as amostras foram recolhidas em recipiente limpo, sem preservativos e guardadas na geladeira, até as $6 \mathrm{~h}$ da manhã. Os pacientes foram ori- entados a não praticar exercícios físicos dois dias antes de iniciar a coleta de urina, não ter relação sexual no dia da coleta, não comer carne em excesso nos dias que antecederam a coleta e as mulheres foram orientadas a não fazer a coleta no período menstrual. Este procedimento foi repetido três vezes com intervalo mínimo de uma semana entre cada coleta em um período máximo (entre a primeira e a última coleta) de seis meses. Os pacientes colheram, no hospital, amostras de urina para a realização de $M$ ultistix ${ }^{\circledR}$ (Bayer, D iagnostics), urinálise e urinocultura para se excluírem outras doenças renais, cetonúria e infecção urinária. As dosagens da concentração urinária de albumina foram realizadas por radioimunoensaio (Diagnostic Product Corporation Los Angeles, com sensibilidade de $0,3 \mu \mathrm{g} / \mathrm{ml}$ e coeficientes de variação intraensaio e interensaio respectivamente de 2,7\% e 3,5\%).

O s pacientes diabéticos e os não diabéticos foram classificados conforme critérios considerados consenso de literatura (21), como normoalbuminúricos quando a taxa de excreção urinária de albumina (EUA) em duas amostras consecutivas era $<20 \mu \mathrm{g} / \mathrm{min}$.

O exame de fundo de olho foi realizado pelo Serviço de Oftalmologia do Hospital U niversitário Pedro Ernesto nos pacientes diabéticos, por oftalmoscopia indireta com o oftalmoscópio "Welch Allyn" sob efeito de medicação midriática tópica. $O$ fundo de olho foi classificado em normal quando ambos os olhos não apresentavam qualquer lesão retiniana.

A doença macrovascular clínica foi excluída pelo questionário da O rganização M undial de Saúde (22) e por eletrocardiograma de repouso de acordo com os critérios estabelecidos por M innesota (23). de fumar.

Consideramos como tabagismo o hábito diário

Foram obtidas amostras de sangue de toda a população estudada após jejum mínimo de 12 horas na visita ao hospital para determinação dos seguintes exames: glicemia de jejum pelo método glicose oxidase; hemo- 
globina glicosilada (H bA1c) por H PLC pelo aparelho Merk H itachi 2.91 .00 (VR: 4,0-6,2\%); fibrinogênio, $\alpha 1-G P A$ foram determinados por turbidimetria e PCR por método ultra-sensível de nefelometria. O s valores de referência (VR) e os coeficientes de variação (CV) intraensaio e interinsaio são respectivamente: $\mathrm{Fi}$ brinogênio (Behring Turbitimer - M arburg, Alemanha; sensibilidade de $35,0 \mathrm{mg} / \mathrm{dl}$ ) VR=180-350 mg/ dl; CV intraensaio $=5,2 \%$ e interensaio $=2,5 \% ; \alpha 1$-GPA Glicoproteína ácida (Behring Turbitimer - M arburg, Alemanha; sensibilidade de 40,0mg/ dl) VR $=40-130 \mathrm{mg} / \mathrm{dl}$; CV intraensaio $=4,1 \%$ e interensaio $=3,2 \%$ PCR (APTEC - Selectra M erck - D armstadt, Alemanha; sensibilidade de $0,01 \mathrm{mg} / \mathrm{dl}) \quad V R=0,0-1,0 \mathrm{mg} / \mathrm{dl} ; \mathrm{CV}$ intraensaio $=2,9 \%$ e interensaio $=2,2 \%$

0 protocolo do estudo foi aprovado pela Comissão de Ética do Hospital U niversitário Pedro Ernesto.

Os dados foram analisados no programa EPIINFO versão 6.0, sendo complementado pelo SPSS (Statistical Packagefor the Social Sciences) para Windows versão $8.0,1992$. Todas as variáveis foram testadas quanto à sua normalidade pelo teste de Shapiro. Os resultados foram apresentados como média \pm desviopadrão para as variáveis com distribuição normal e como mediana (mínimo e máximo) para as variáveis sem distribuição normal. O s seguintes testes estatísticos foram utilizados: Teste $t$ para comparação de duas médias quando a variável em análise apresentava distribuição normal e teste não paramétrico de $M$ ann-Whitney $(Z)$ quando a distribuição não era normal. Foram realizadas correlações de Pearson e Spearman para avaliar o grau de correlação entre as variáveis contínuas. A regressão múltipla em stepwise foi realizada após ajuste para idade, sexo, tabagismo e I M C para análise de correlações entre três ou mais variáveis contínuas selecionadas quando apresentavam $p<0,10$ na correlação de Pearson. N esta análise, as variáveis dependentes sem distribuição normal entraram no modelo após transformação logarítmica. Q uando indicado, apresentamos o limite de confiança de $95 \%$ ( $95 \% \mathrm{CL}$ ). Consideramos como significativo um valor de $p$ bi-caudal $\varangle 0,05$.

\section{RESULTADOS}

$\mathrm{N}$ a comparação entre os grupos de diabéticos e não diabéticos, observamos que, das proteínas de fase aguda estudadas, a proteína $C$ reativa $[0,23(0,01$ $2,90)$ vs. $0,14(0,01-2,41) \mathrm{mg} / \mathrm{dl} ; \mathrm{p}=0,0172]$ e a alfa 1 glicoproteína ácida [53,5 (37,0-115,0) vs. 40,0 $(19,0-8,0) \mathrm{mg} / \mathrm{dl} ; \mathrm{p}<0,0001]$ mostraram-se com níveis superiores nos pacientes diabéticos. $\mathrm{N}$ ão houve diferença em relação aos níveis de fibrinogênio. O s indivíduos não diabéticos apresentaram maior IMC que os diabéticos, respectivamente $(22,1 \pm 3,7$ vs. $\left.20,4 \pm 2,9 \mathrm{~kg} / \mathrm{m}^{2} ; p=0,01\right)$. Não houve diferença em relação aos níveis de pressão arterial sistólica e diastólica e na freqüência de tabagismo. Estes dados estão descritos na tabela 2.

Tabela 2. Comparação dos grupos não diabéticose diabéticos.

\begin{tabular}{lcccc}
\hline Variáveis & $\begin{array}{c}\text { Não Diabéticos } \\
\mathrm{n}=66\end{array}$ & $\begin{array}{c}\text { Diabéticos } \\
\mathrm{n}=48\end{array}$ & $\begin{array}{c}\text { Teste } \\
\text { Estatístico }\end{array}$ & Valor de $\mathrm{p}$ \\
\hline PAS (mmHg) & 106,3 & 107,6 & $\mathrm{Z}=1,093$ & 0,2958 \\
PAD (mmHg) & $(90,0-130,7)$ & $(96,0-133,0)$ & $\mathrm{Z}=1,346$ & 0,2458 \\
IMC (Kg/m²) & 68,3 & 70,3 & 0,0142 \\
Glicemia (mg/dl) & $(52,6-82,6)$ & $(56,0-91,3)$ & $\mathrm{t}=2,4887$ & 0,45 \\
HbAlc (\%) & $22,1 \pm 3,7$ & $20,4 \pm 2,9$ & & 0.9587 \\
Tabagismo(S/N) & 78,7 & 176,0 & \\
Fibrinogênio (mg/dl) & $(64,0-97,0)$ & $(59,0-489,0)$ & & $0,0172 *$ \\
PCR (mg/dl) & $3,28 \pm 0,45$ & $8,25 \pm 2,14$ & & $<0.0001^{*}$ \\
Alfa 1 (mg/dl) & 210,0 & $4 / 44$ & $\mathrm{Z}=0,003$ & $\mathrm{Z}=5,667$ \\
\end{tabular}

*: p com signific a do estatístic o

PAS: pressão arterial sistólic a; PAD: pressã o arterial diastólic a; PCR: proteína C reativa;

Alfa 1: alfa glicoproteína ácida. 
Considerando-se as proteínas de fase aguda, observamos, na população geral estudada, correlação entre $\alpha 1$-GPA e PCR $(r=0,28 ; p=0,004)$.

$\mathrm{N}$ a população geral estudada, observamos correlação entre a $\alpha 1$-GPA e H balc $(r=0,53 ; p=0,000)$ e glicemia $(r=0,57 ; p=0,002)$; não havendo correlação com as demais variáveis analisadas. $\mathrm{Na}$ análise de regressão múltipla em stepwise com a $\alpha \mathrm{l}$-GPA como variável dependente, após ajuste por sexo, idade, IM C e tabagismo, as variáveis independentes significativas foram de acordo com a ordem de entrada no modelo: a $\mathrm{H}$ bAlc $r=0,53 ; r^{2}=0,26$ [95\% CL $(0,002-0,02) ; B=$ $0,01 ; p=0,000]$ e a glicemia $r=0,57 ; r^{2}=0,29[95 \% C L$ $(4,6 \mathrm{E}-05$ a 7,1 E-04); $B=3,7 \mathrm{E}-04 ; \mathrm{p}=0,000]$. O bservamos correlação entre PCR eH bA $1 c(r=0,18 ; p=0,05)$ e tendência com a glicemia $(r=0,16 ; p=0,07)$, não havendo correlação com as demais variáveis analisadas. $\mathrm{N}$ a análise de regressão múltipla em stepwise com a PCR como variável dependente, nenhuma variável independente foi significativa. O bservamos tendência à correlação entre fibrinogênio e IM C $(r=0,17 ; p=0,07)$, não havendo correlação com as demais variáveis analisadas.

$\mathrm{Na}$ análise intra-grupo das proteínas de fase aguda estudadas não observamos correlação entre a1GPA e glicemia e H bAlc no grupo de diabéticos; no grupo controle observamos correlação entre fibrinogênio e IMC $(r=0,25 ; p=0,041)$ e HbAlc $(r=$ $0,25 ; p=0,044)$.

\section{DISCUSSÃO}

O s diabéticos podem apresentar níveis elevados das proteínas de fase aguda, como a proteína $C$ reativa, a alfa 1 glicoproteína ácida e o fibrinogênio, cujo significado difere dependendo do estágio da doença. No início da doença, a reação inflamatória resulta em um aumento de citoquinas, incluindo o fator de necrose tumoral a (TNF- $\alpha$ ), a interleucina $1 \beta$ (IL1 $\beta$ ) e a interleucina 6 (IL 6), que apresentam uma inter-relação com as proteínas de fase aguda (24). Com a evolução da doença, a persistência destas proteínas em níveis acima do normal representaria um estado de inflamação crônica leve, que poderia ser um dos fatores responsáveis pela aterosclerose acelerada desta população $(2,25)$. Recentemente, foi demonstrado aumento da PCR em pacientes diabéticos tipo 1, associado a uma maior espessura da camada íntima endotelial, que é considerado um indicador da aterosclerose pré clínica (24).

A proteína $C$ reativa aumenta em resposta a vários estímulos e seu papel como um marcador de risco para o desenvolvimento de doença cardiovascular tem sido investigado em pacientes diabéticos e em indivíduos saudáveis (26).

A alfa 1 glicoproteína ácida também pode estar aumentada nos pacientes diabéticos como um marcador da inflamação, seu papel ainda não está bem definido, mas sabe-se que envolve regulação da resposta imune (8). R. Guillot e cols. (27) descreveram, recentemente, que a $\alpha 1$-GPA seria necessária para a manutenção da permeabilidade capilar normal no mesentério e nos glomérulos renais. É relatado que os níveis da $\alpha$ l-GPA têm relação com a idade, o sexo, com a dor crônica, estado nutricional e doença (28). U m estudo realizado com 44 diabéticos do tipo 2 demonstrou um aumento da $\alpha$ l-G PA nestes pacientes, principalmente quando apresentavam síndrome metabólica, comparados ao controle (29). O utros estudos também demonstraram aumento da $\alpha 1-\mathrm{GPA}$ em pacientes diabéticos tipo $2(3,16)$, sendo que em um deles este aumento foi preditor da mortalidade cardiovascular (16). Recentemente, foi demonstrado que altos níveis de $\alpha 1-$ GPA foram preditores de evolução para o diabetes tipo 2 (30).

É descrito na literatura, por diversos estudos epidemiológicos, o papel do fibrinogênio plasmático como um fator de risco independente para o infarto agudo do miocárdio e o acidente vascular cerebral na população em geral $(31,32)$.

Em nosso estudo, não houve diferença nos níveis plasmáticos de fibrinogênio entre os diabéticos e os não diabéticos. El K hawand e cols. (33) também não observaram níveis de fibrinogênio aumentados em pacientes diabéticos quando comparados aos indivíduos não diabéticos. No estudo desenvolvido por Gnada e Arkin (9), os níveis de fibrinogênio estavam levemente aumentados nos diabéticos tipo 1 em comparação ao controle, mas o principal fator responsável por este aumento foi a presença concomitante de complicações vasculares. 0 diabetes, como fator independente, é minimamente responsável pelos níveis de fibrinogênio aumentados que podem ocorrer nos pacientes acometidos por esta patologia (34). Vários fatores como a doença vascular, a idade, o fumo e a obesidade (35), e ainda a hiperglicemia e o hiperinsulinismo (36-38) talvez tenham um papel importante nos níveis do fibrinogênio e devem também ser analisados. Como a maioria dos fatores associados aos níveis elevados de fibrinogênio descritos em outros estudos (obesidade, idade mais avançada, complicações vasculares) não estava presente nos pacientes diabéticos por nós estudados e, como o fumo era pareado com o grupo de indivíduos não diabéticos, isso poderia justificar a ausência das alterações nas 
concentrações do fibrinogênio em nosso estudo. J ensen e cols. (39) reportaram um aumento progressivo nos níveis de fibrinogênio com 0 aumento da severidade da proteinúria. Greaves e cols. (40) não encontraram diferença entre os diabéticos tipo 1 normoalbuminúricos e microalbuminúricos em relação aos níveis do fibrinogêrnio, mas observaram que os pacientes com EUA $>200 \mu \mathrm{g} / \mathrm{min}$ apresentavam maiores concentrações.

Em relação às outras duas proteínas de fase aguda dosadas em nosso estudo, houve uma diferença significativa entre os grupos de diabéticos e não diabéticos, sendo evidenciado que os diabéticos apresentaram níveis maiores tanto da PCR, quanto da $\alpha 1-G P A$.

As concentrações plasmáticas da PCR são determinadas apenas por sua taxa de produção (41), sugerindo que seu aumento nos diabéticos tipo 1 não tenha relação com alterações na função renal que estes pacientes possam apresentar.

U m estudo de caso-controle com 40 pacientes portadores de diabetes tipo 1, não fumantes e sem complicações macrovasculares (2), demonstrou um aumento da PCR nos diabéticos em relação ao controle, mesmo após a exclusão dos 10 pacientes com microalbuminúria. Este estudo ainda mostrou uma associação entre concentrações elevadas da PCR e ativação endotelial, avaliada pelo fator de von Willebrand, sugerindo que o aumento da PCR nos diabéticos tipo 1 não seja causado pelo desenvolvimento ou pela presença de complicações microvasculares, mas talvez preceda o aparecimento desta complicação.

U ma série de estudos demonstram uma associação $(5,26,42)$ e uma correlação $(6,43)$ positiva entre a PCR e o IM C, evidenciando que os obesos têm concentrações mais elevadas desta proteína, o que suporta a idéia de que um estado de inflamação sistêmica leve e crônica esteja presente na obesidade (44). E m nosso estudo, não observamos tal correlação, possivelmente devido ao fato dos pacientes diabéticos terem apresentado uma média de I M C menor que 25.

Relativamente, poucos estudos sobre a associação da PCR e diabetes tipo 1 têm sido publicados. A PCR tem-se mostrado com maiores concentrações em pacientes diabéticos tipo 2 ou com intolerância à glicose que em indivíduos controle (15). D evido ao fato dos pacientes diabéticos apresentarem um risco aumentado de doença cardiovascular, o aumento nos níveis da PCR poderia refletir, em parte, o componente inflamatório do processo aterosclerótico, tão prevalente entre os indivíduos acometidos pelo diabetes. E ainda, uma resposta inflamatória talvez possa ser desencadeada dentro dos mecanismos fisiopatológicos do diabetes.
Vários mecanismos possíveis poderiam induzir um estado inflamatório crônico e moderado no diabetes, representado pelo aumento das proteínas de fase aguda, um dos principais seria a baixa insulinemia no sistema porta, o que propiciaria um aumento da produção destas proteínas pelo fígado, que normalmente é inibida pela insulina (45). O utros mecanismos incluem 0 aumento do stress oxidativo induzido pela hiperglicemia, ativação de macrófagos e a indução de citocinas (2). U ma das conseqüências fisiopatológicas da hiperglicemia é o fenômeno de glicação não enzimática e a formação de produtos finais da glicação avançada (46), estes produtos podem ativar os macrófagos, aumentar o stress oxidativo (2) e induzir nos macrófagos a síntese de interleucina-1 e do fator de necrose tumoral-a, que estão relacionados ao aumento de algumas proteínas de fase aguda (47). U ma outra possibilidade seria que 0 aumento nos níveis da PCR estaria relacionado às citocinas derivadas do tecido adiposo visceral e subcutâneo abdominal (48), como a interleucina-6, que estimularia a produção desta proteína pelo fígado (49), reforçando o que já foi discutido a respeito da associação entre obesidade e PCR, mas que, provavelmente, não ocorre em diabéticos tipo 1, predominantemente magros, conforme nossa amostra. $\mathrm{No}$ nosso estudo, os indivíduos não diabéticos tiveram maior peso e menores níveis de PCR e $\alpha 1-G P A$, ratificando que possivelmente o principal mecanismo seja 0 stress oxidativo induzido pela hiperglicemia.

$\mathrm{Na}$ análise multivariada na população geral, as variáveis independentes preditivas e associadas à a1GPA foram a H bA 1c e a glicemia, que explicaram $29 \%$ da variabilidade desta proteína de fase aguda.

U ma das limitações do nosso estudo foi o pequeno número de pacientes e o uso do questionário da OMS e do código de M innesota de 12 derivações no ECG de repouso para diagnóstico de doença cardiovascular clínica. Embora todos os pacientes com possível doença cardiovascular tenham sido excluídos do estudo, há possibilidade de que pacientes com processo ateroesclerótico préclínico tenham sido incluídos. Recentemente, diferentes estudos em pacientes jovens com diabetes mellitus tipo 1 demonstraram que altos níveis de PCR estiveram correlacionados com espessamento da camada íntima-média da carótida, um índice subclínico da aterosclerose inicial desta artéria (25), e associado com calcificação das artérias coronárias, um índice de aterosclerose coronariana (50). A mbos os estudos mostraram que altos níveis de PCR foi um fator de risco independente da presença do Diabetes tipol. 
Além disso, devido ao desenho do nosso estudo e de nossa amostra ser constituída de pacientes sem complicações crônicas, nós não podemos estabelecer se há uma relação causal e temporal entre proteínas de fase aguda e microalbuminúria e/ ou retinopatia. Entretanto, um estudo prospectivo recente com pacientes com diabetes tipo 2 demonstrou que a microalbuminúria foi, entre outros fatores, preditora de altos níveis de $P C R$, fibrinogênio e marcadores da função endotelial, sugerindo uma inter-relação entre função endotelial, inflamação crônica e microalbuminúria (51).

Concluímos que o aumento das proteínas de fase aguda, em nosso trabalho representadas pela alfa 1 glicoproteína ácida e pela proteína C reativa, ocorreu nos diabéticos tipo 1 normoalbuminúricos, sem retinopatia e sem doença macrovascular clínica. Estudos prospectivos poderão determinar se este estado de inflamação crônica, que provavelmente existe nestes pacientes, estaria relacionado ao processo de aterosclerose acelerado e ao desenvolvimento das complicações crônicas do diabetes.

\section{AGRADECIMENTOS}

Agradecemos ao apoio da FAPERJ, processo número E26/ 171605199.

\section{REFERÊNCIAS}

1. Kannel WB, McGee DL. Diabetes and cardiovascular disease. The Framingham study. JAMA 1979;241:2035-8.

2. Schalkwijk CG, Poland DCW, VanDijk W, KokA, EmeisJJ, Dräger AM, et al. Plasma concentration of C-reactive protein is increased in type 1 diabetic patients without clinical macroangiopathy and correlates with markers of endotelial dysfunction: evidence for chronic inflammation. Diabetologia 1999;42:351-7.

3. Ebeling P, Teppo AM, Ko istinen HA, Vikari J, Rönnema a $T$, Nissen $M$, et al. Troglitazone reduces hyperglycaemia and selectively acute-phase serum proteins in patients with type II diabetes. Diabetologia 1999;42:1433-8.

4. Yu H, Rifai N. High-sensitivity $\mathrm{C}$-reactive protein and atherosclerosis: from theory to therapy. Clin Biochem 2000;33:601-10.

5. Cook GD, Mendall MA, Whincup PH, Ca rey IM, Ballam L, Momis JE, et al. C-reactive protein concentration in children: relationship to adiposity and other cardiovascular risk factors. Atherosclerosis 2000;149:139-50.

6. Haverkate F, Thompson SG, Pyke SDM, Gallimore JR, Pepys MM. Production of $C$-reactive protein and risk of coronary events in stable and unstable angina. Lancet 1997;349:462-6.
7. Ridker PM, Cushman M, Stampfer MJ, Tracy RP, Hennekens $\mathrm{CH}$. Inflammation, aspinin and the risk of cardiovascular disease in apparently healthy men. $\mathrm{N}$ Engl J Med 1997;336:973-9.

8. Kamori T, Nonaka A, Maruyama T, Otagiri M. Effect of clarithromyc in on al-acid glycoprotein levels in nomal and diabetic rats. Res Comm Mol Path Pharm 1998;101:233-40.

9. Ganda OP, Aarkin CF. Hyperfibrinogenemia. An important risk factor for vascular complications in diabetes. Diabetes Care 1992;15:1245-50.

10. Emst E, Resch KL. Fibrinogen as a Cardiovascular Risk Factor: A Meta-Analysis and Review of the Literature. Ann Intem Med 1993;118:956-63.

11. Ceriello A, Pirisi M, Giacomello R, Stel G, Falleti E, Motz E, et al. Fibrinogen plasma levels as a marker of thrombin activation: new insights on the role of fibrinogen as a cardiovascular risk factor. Thromb Haem 1994;71(5):593-5.

12. Ceriello A, Taboga C, Giacomello R, Falleti, Stasio GE, Motz E, et al Fibrinogen plasma levels as a marker of thromb in a ctivator in dia betes. Diabetes 1994;43:430-2.

13. Ceriello A. Coagulation activation in diabetes mellitus: the role of hyperglycaemia and therapeutic prospects. Diabetologia 1993;36:1119-25.

14. Ceriello A, Mercuri F, Fabrro D, Giacomello R, Stel G, Taboga C, et al. Effect of intensive glycaemic control on fibrinogen plasma concentrations in patients with type II diabetes mellitus. Relation with b-fibrinogen genotype. Diabetologia 1998;41:1270-3.

15. Mc Millan DE. Increased levels of a cute-phase proteins in diabetes. Metabolism 1989;38(11):1042-6.

16. Christiansen MS, Hommel E, Magid E, Rasmussen BF. Orosomucoid in urine predicts cardiovascular and over-all mortality in patients with Type II diabetes. Diabetologia 2002;45:115-20.

17. The Expert Committee on The Diagnosis and Classification of Diabetes Mellitus. Report the expert committee on the diagnosis and classification of diabetes mellitus. Diabetes Care 1997;20:1183-97.

18. Tanner JM. Growth at adolescence. $2^{\text {nd }}$ ed. Oxford: Blac kwell, 1962.

19. National High Blood Pressure Education Program Working Group on Hypertension Control in Children and Adolescents Update on the 1987 task force report on high blood pressure in children and adolescents: a working group report from the national high blood pressure education program. Pediatrics 1996;88(4):649-58.

20. The Sixth Report of The Joint National Committee on Detection, Evaluation and Treatment of High Blood Pressure. Arch Intern Med 1997;157:2413-45.

21. Garber AJ . (Chair) Consensus development conference on the diagnosis and management of nephropathy in patients with diabetes mellitus. Diabetes Care 1994;17:1357-61.

22. Rose GA, Blackbum H, Gillum RF, Phineas RJ . Cardiovascular Survey Methods. WHO Monograph Series 1982;(56):162-5.

23. Blackbum H, Keys A, Simonson E, Rautaharju P, Punsar S. The electrocardiogram in population studies: a classification system. Circulation 1960;(21):217-33. 
24. Romano $M$, Pomilio $M$, Vigneri S, Falco A, Chiesa PL, $C$ hia relli $F$, et al. End othelial Perturbation in Child ren and Adolescents with Type 1 Diabetes. Diabetes Care 2001;24(9):1674-8.

25. Haya ishi-Okano R, Yamasaki Y, Katakami N, Ohtoshi K, Gorogawa SS, Kuroda A, et al. Elevated C-Reactive Protein Associates with Early-Stage Carotid Atherosclerosis in Young Subjects with Type 1 Diabetes. Diabetes Care 2002;25(8):1432-8.

26. Ford ES. Body mass index, diabetes, and C-reactive protein a mong US a dults. Diabetes Care 1999;22(12):1971-7.

27. Guillot R, Kassab JP, Ogneva V, André J, Durussel JJ, Hadjinsky P, et al. Relation between pancreatic islet cellular infiltration and plasma fibrinogen or al-acid glycoprotein levels in spontaneously and streptozotocin-diabetic rats: an increase in these protein levels is not necessary for inducing mic rocirc ula tory erythrocyte velocity alteration. Pancreas 1994;9(3):336-43.

28. Valle $M$, Esteban M, Rodriguez-Sasia in JM, Calvo $R$, Aguirre C. Characteristics of serum protein binding of felodipine. Res Comm Mol Path Pharm 1996;94:73-87.

29. Pickup J C, Mattock MB, Chusney GD, Burt D. NIDDM as a disease of the innate immune system: association of acute-phase reactants and interleukin- 6 with metabolic syndrome X. Diabetologia 1997;40:1286-92.

30. Schimidt MI, Tracy RP, Heiss G for the ARIC investigators. Markers of inflammation and prediction of diabetes mellitus in adults (Atherosclerosis risk in communities study): a cohort study. Lancet 1999;353:1649-52.

31. Wilhelmsen L, Svardsudd K, Korsan-Bengtsen K. Fibrinogen as a risk factor for stroke and myocardial infarction. N Engl J Med 1984;311:501-5.

32. Emst E. Plasma fibrinogen - an independent cardiovascular risk factor. J Intern Med 1990;227:365-72.

33. El Khawand C, Lavenne E, Jamart J. Hemostasis variables in type I diabetic patients without demonstrable vascular complic ations. Diabetes Care 1993;16:1137-45.

34. Vague $P$, J uhan-Vague I. Fibrinogen, fibrinolysis and diabetes mellitus: a comment. Diabetologia 1997;40:738-40.

35. Lowe GDO. The impact of fibrinogen on arterial disease. Topics in Preventive Cardiology. Excerpta Medica 1993;3-11.

36. Meigs J B, Mittleman MA, Nathan DM, Tofler GH, Singer $D E$, Murphy-Sheehy PM, et al. Hyperinsulinemia, hyperglicemia, and impaired hemostasis. The Framingham offspring study. J AMA 2000;283(2):221-8.

37. Eliasson M, Evrin PE, Roder ME, Lindahl B, Dinesen B. Proinsulin, intact insulin, and fibrinolytic variables and fibrinogen in healthy subjects. Diabetes Care 1997;20:1252-4.

38. Emanuele N, Azad N, Abraira C, Henderson W, Colwell $J$, Levin S, et al. Effect of intensive glycemic control on fibrinogen, lipids, and lipoproteins. Arch Intem Med 1998;158:2485-90.

39. J ensen T, Stender S, Deckert T. Abnomalities in plasma concentration of lipoproteins and fibrinogen in type 1 (insulin-dependent) diabetic patients with increase urinary a lbumin Diabetologia 1988;31:142-5.
40. Greaves M, Malia RG, Goodfellow K, Mattock M, Stevens LK, Stephenson JM, et al, and The Eurodiab IDDM Complications Study Group. Fibrinogen and von Willebrand factor in IDDM: Relationships to lipid vascular risk factors, blood pressure, glycaemic control and urinary albumin excretion rate: the EURODIAB IDDM complication study. Diabetologia 1997;40:698-705.

41. Vigushin DM, Pepys MB, Hawkins PN. Metabolic and scintigraphic studies of radioiodinated human $C$-reactive protein in health and disease. J Clin Invest 1993;91:1351-57.

42. Koening $W$, Sund $M$, Fröhlich $M$, Fischer $H G$, Löwel $H$, Döring A, et al. C-reactive protein, a sensitive marker of inflammation, predicts future risk of coronary heart disease in initially healthy middle-aged men. Results from the MONICA (Monitoring Trends and Deteminants in Cardiova scular Disease) Augsburg Cohort Study, 1984 to 1992. Circulation 1999;99:237-42.

43. Mendall MA, Patel P, Ba llam L, Stra chan D, Northfield TC. $C$ reactive protein and its relation to cardiovascular risk factor: a population based cross sectional study. BMJ 1996;312:1061-5.

44. Visser M, Bouter LM, McQuillan GM, Wener MH, Ha mis TB. Elevated C-reactive protein levels in overweight and obese adults. J AMA 1999;282(22):2131-5.

45. Chen J W, J ensen J S, Gall MA, Deckert M, Yokoyama H, Parving $\mathrm{HH}$. Raised serum sialic acid concentration in NIDDM patients with and without diabetic nephropathy. Diabetes Care 1996;19:130-4.

46. Vlassara H, Bucala R, Striker L. Pathogenic effects of advanced glycosylation: biochemical, biological, and clinical implications for diabetes and aging. Lab Invest 1994;70:138-51.

47. Vlassara H, Brownlee M, Manogue HR, Dinarello C, Pasagian A. Cachetin/TNF and IL-1 induced by glucose modified proteins: role in nomal tissue remodeling. Science 1988;240:1546-8.

48. Hotamisligil GS, Amer P, Caro JF, Atkinson RL, Spiegelman BM. Increased adipose tissue expression of tumor necrosis factor-alpha in human obesity and insulin resistance. J Clin Invest 1995;95:2409-15.

49. Banks RE, Forbes MA, Storr $M$. The acute phase response in patients receiving subcutaneous IL-6. Clin Exp Immunol 1995;102:217-23.

50. Colhoun HM, Schalkwijk C, Rubens MB, Stehouwer CDA. C-reactive protein in type 1 diabetes and its relationship to coronary artery calcification. Diabetes Care 2002;25:1813-7.

51. Stehouwer CDA, Gall MA, Twisk JWR, Knudsen E, Emeis JJ, Parving $\mathrm{HH}$. Increased urinary albumin excretion, endothelial dysfunction, and chronic low-grade inflammation in type 2 diabetes. Diabetes 2002;51:1157-65.

Endereço para correspondência:

La ura J a bur Pic c irillo

Av Sete de Setembro 2306, apto. 302B

40080-001 Salva dor, BA 